Aus dem pathologisch-anatomisehen Institut der Universitat Leipzig.

(Direktor: Gel.. Rat Prof. Dr. Marchand.)

\title{
Fall von Missbildung des Herzens und der Gefässe.
}

\author{
Von \\ Dr. med. Karl Jaffe, \\ approb. Arzt aus Leipzig.
}

Mit 2 Abbildungen.

Der in der vorliegenden Arbeit behandelte Fall stellt infolge des Zusammentreffens mehrerer, zum Teil nicht häufig beobachteter Missbildungen eine Seltenheit dar. Er betrifft ein 22 jähriges Dienstmädchen und lässt Missbildungen des Herzens, der grossen Arterien und der grossen Venen neben kongenitalen Anomalien der Baucheingeweide erkennen. Eine kurze vorläufige Mitteilung des vorliegenden Falles ist bereits durch Prof. Herzog in der medizinischen Gesellschaft zu Leipzig (Münchener medizinische Wochenschrift 1913, Nr. 37, S. 2090) erfolgt, daselbst hat auch Dr. Mo hr über den klinischen Verlauf des Falles berichtet. Es sei mir gestattet, im folgenden diesen Fall, sowie die Beziehungen, die sich durch Vergleich mit den bisher in der Literatur beobachteten ähnlichen Fällen ergeben, eingehender zu behandeln.

Nach der Krankengeschichte, deren Überlassung ich Herrn Geheimrat von Strümpell und Herrn Prof. Dr. Assmann verdanke, konnte die Patientin über den Beginn ihrer Erkrankung nichts Bestimmtes aussagen, glaubte sich jedoch zu erinnern, dass sie schon seit früher Kindheit nicht imstande war, rasch zu laufen oder körperliche Anstrengungen auszubalten. Seit Dezember 1912 litt sie an allgemeiner Schwäche, Atemnot beim frehen, 
Herzklopfen und Schwindelmüälen, war jedoch noch fähig, ihren Beruf auszuïben. Am 'lage ihrer Entlassung aus dem Krankenhaus ist die Patientin plötzlich beim Hinaufsteigen in ihre vier Treppen hoch gelegene Wohnang collabiert und verstorben.

Die Sektion der Leiche (Matha M., 22jühriges Dienstmädeben, gest. am 8. V11. 13., Leichennummer 1115/1913) wurde am 'T'age nach dem Tode auf dem Friedhof von Prof. Herzog vorgenommen, wobei die Organe der Brust- und Bauchböhle in toto entfernt worden. Nachträglich wurde eine Injektion ter Arterien und Venen ausefuthrt.

Mittelgrosse jugendliche weibliche Leiche von etwas grazilem Körperbau in gutem Ernährungszustand. Die Skelettmuskulatur ist in müssigem Grade entwickelt. Die Haut des Gesichts ist blaurot verfärbt. Die Totenflecke sind mässig dunkel an Rücken. Ödeme bestehen nirgends. Das linke Bein ist gegenüber dem rechten um etwa 5 Querfinger verkürzt, seine Muskulatur ist etwas schwicher ausgebildet als rechts. Der Trochanter major steht linkerseits 4 bis 5 Querfinger über der Roser-Nelat on schen linie. Die Behaarung des Kopfes ist nur geriug, die Mammile sind ziemlich dürftig entwickelt.

Bei Eröffinung des Thorax liegt der enorm ausgedelınte Herzbeutel frei vor. Die Lungen verschwinden fast völlig in den Pleuraböhlen. Die Spitze des Herzbeutels berïhrt fast die linke seitliche Brustwand, und auch rechts reicht das Herz 3 bis 4 Querfinger über die Mittellinie hinaus. In der liuken Pleurahüble finden sich 100 bis $200 \mathrm{ccm}$, in Herzbeutel nur wenige Kubikzentimeter einer klaren hellgelben Flüssigkeit. Zwerchfellstand links 6. Rippe, rechts 5. Intercostaliaum.

Dus $\mathrm{Herz}$ ist enorm vergrössert. Der rechte Ventrikel ist allein fast doppelt so gross als die Faust der teiche und bildet allein die Spitze (rgl. Abbildung 1). Der rechte Vorhof ist gleichfalls etwa faustgross, fühlt sich selnr dickwandig an und springt nach rechts stark vor. Der linke Ventrikel kommt, von vorn betrachtet, kaum zur Ansicht, bildet gleichsam uur einen Anhavg des Herzens, und ebenso ist von vorn vom linken Vorhof nur eben die Spitze des kleinen Herzobrs zu sehen. Die Länge des Herzens beträgt - an der Vorderfläche vom Ostium arteriosum dextrum bis zur Spitze gemessen - $16 \mathrm{~cm}$, der grösste Durchmesser am Herzkranz 13,5 cm. Die Pulnonalis ist enorm erweitert und prall gefullt, etwa fünf Querfinger dick (im Durchmesser $3,5-4 \mathrm{~cm}$ ); der Conus pulmonalis ist dick und etwas vorgewölbt, so dass er in der Herzsilhouette als obere linke Begrenzung hervortritt; nur ein kleiner Teil der linken Herzgrenze wird - bei der Ansicht von vorn - vom linken Ventrikel gebildet. Dagegen ist besonders auffallend das geringe Volumen der Aorta, die nur etwa daumendick (2 cm im Durchmesser) ist.

Am gehärteten Praparat werden Ventrikel und Vorhöfe eröffnet. Sieht man in den rechten Vorhof hinein, so liegt ein äber 5-Markstück grosser Defekt in unteren 'l'eil des Vorbol'septuns vor; das letztere bildet dabei eine uach unten ziemlich scharf begrenzte halbmondförmige Falte. Im Vorhofseptum ist noch ein weiterer', höher gelegener, von einem fädigen 
fibrösen Gitterwerk überkreuzter kreisrunder Defekt vorhanden, der seiner Lage nach dem Foramen ovale entspricht. Durch den erstgenannten Defekt, der die beiden Vorhöfo miteinander verbindet, gelangt man mit dem Finger ohne weiteres einerseits in die Mitralis, andererseits in die Tricuspidalis.

Das.Septum ventriculorum ist verkürzt und nach oben halbmondförmig eingesunken, etwa $7 \frac{1}{2} \mathrm{~cm}$ hoch. Von dem First des Ventrikelseptums, der eigentümliche, besonders nach rechts hinüberhängende grobknollige Verdickungen trägt, entspringt nach links der Aortenzipfel der Mitralis und nach rechts das verkümmerte mediale Tricuspidalsegel; letzteres stellt nur einzelne knopffürmig vorspringende derbe weissliche Verdickungen dar. Der Aortenzipfel der Mitralis ist in zwei Teile, einen vorderen und einen hinteren gespalten. Der vordere Papillarmuskel der Tricuspidalis ist enorm verdickt; das vordere Tricuspidalsegel ist sehr gross und stark entwickelt, im ganzen $6 \mathrm{~cm}$ breit; das hintere Segel geht nach unten nur teilweise in regelrechte Sehnenfäden ubber, der mediale Teil laụft in ein Netzwerk von derben Strängen aus, das mit einigen zarten Verbindungen an der Herzwand medial und hinten ansetzt.

Die Höhle des rechten Ventrikels ist stark dilatiert, seine Muskulatur sehr hypertrophisch. Letztere misst am gehärteten Präparat im Bereich des Conus pulmonalis ohne Papillarmaskeln $6 \mathrm{~mm}$, doch hat hier die mehrfach in dicken Balken entwickelte trabekuläre Schicht den grösseren Anteil an der Hypertrophic. An der rechten Kante misst die Herzwand einschliesslich trabeknlärer Schicht 1,2 bis $1.3 \mathrm{~cm}$; ohne dieselbe $8 \mathrm{~mm}$. Das Lumen des linken $V$ entrikels ist ganz plattgedrückt, seine Muskulatur misst ohne trabekuläre Schicht $7 \mathrm{~mm}$. Die Mitralis ist für $1 \frac{1}{2}$. Finger durchgängig. Grosse Gefïsse: D'r Bogen der Aorta übersteigt den rechten Hauptbronchus; die Aorta verläuft an der rechten Seite der Wirbelsüule bis 2ur 'Teilungsstelle in die Arteriae iliacae. Am Aruns gibt die Aorta isoljert zunachst die Arteria carotis sinistra, dann die Arteria carotis dextra und die Arteria subclavia dextra ab. Etwa $1^{1 / 2}$ Querfinger unterhalb der Abgangsstelle der rechten Arteria subclavia, da, wo der Arcus in die Aorta descendens übergeht, zweigt nach links oben gabelförmig hinter dem Ösophagus ein fingerdicker Stamm ab (vgl. Abb. 2, A. a. s.), der sich nach etwa $3 \mathrm{~cm}$ langem Verlauf nach vorn teilt: einerseits in den bleistiftdicken, für eine starke Sonde durchgängigen, an der Arteria pulmonalis haftenden fibrösen Strang des Ligamentum Botalli, andererseits zweigt nach links die Arteria subclavia sinistra ab (vgl. Abb. 2, D. B. und A. s. s.).

Die Vena cava inferior fehlt völlig. In den rechten Vorbof dringt von unten her lediglich die Lebervene ein. Die Valvala Eustacbii ist nur andeutungsweise in Form eines flachen Wulstes vorhanden. Von oben mündet in den rechten Vorhof eine $V$ ena cava superior, in die sich von rechts hinten her die Vena azygos einsenkt; die Vena anonyma fehlt. Es geht nur von der Vena cava superior ein schwaches Gefäss herüber zu der etwas vergrösserten, doppellappigen, etwa 7-8 cm langen und ziemlich fleischigen graurötlichen 'Thymusdrüse. Ein woiterer' etwas höher abgehender Ast verläuft zum Isthmus der Schilddrüse.

Symmetrisch zur Vena cava superior dextra verläuft an der linken 
Seite der Wirbelsäule ein daumendickes Gefäss, das sich aus Vena jugularis sinistra und Vena subelavia sinistra zasammensetzt. In dieses Gefüss mündet ein über den linken Hauptbronchus hinwegsteigendes, daumendickes Gefäss von hinten her ein, das an der linken Seite der Wirbelsäule heraufsteigt (Vena cardinalis inferior sinistra, vgl. Abb. 2 V. c. s.). Der ans dieser Vereinigung resultierende, etwa zwei Querfinger starke Gefässstamm tritt durch den Herzbeutel hindurch, Isuft vor den Lungenvenen an der linken Seite des linken Vorbofs herab und setzt sich in den für 2-3 Finger bequem durchgängigen Sinus coronarius cordis fort, der sich an normaler Stelle mit einem für drei Funger durehgängigen Lumen in den rechten Forhof iffnet. Die Valvula Thebesii ist lang ausgezogen.

Das eben erwähnte daumendicke Gefäss (Vena cardinalis inferior sinistr $\Omega$ ) läuft an der linken Seite der Wirbelsäule abwarts (vgl. Abb. 2, V. c. s.). Die Art seines Durchtritts durch das $Z$ werchfell lässt sich am Präparat vicht mehr feststellen. In der Höhe der linken Niere nimmt es die linke Nierenvene auf. Ltwa einen Querfinger tiefer zweigt von ihm im rechten Winkel eine daumendicke, linter der Aorta nach rechts und etwas schrag nach abwärts verlaufende Querverbindung $a b$, die sich rechts nehen der Aorta teilt: einerseits in dio rechte Nierenvene, andererseits in ein etwa fingerdickes Gefäss (Abb. 2, V. c. d.), das an Stelle der sonst hier verlanfenden Vena cava inferior nach unten zieht und sich bald dem Verlauf der rechten Arteria iliaca communis anschliesst. Die rechte Nierenvene nimmt von unten her noch ein kleines Gefiiss auf, das der Vena spermatica interna dextra entspricht.

Die links neben der Wirbelsäule verlaufende Kardinalvene nimmt etwa 2 Querfinger unterhalb des Abgangs der linken Nierenvene von anten her ein schwaches, der Vena spermatica interna sinistra entsprechendes Gefäss auf (Abb. 2, V. s. s.). Dann schliesst sie sich nach links unten hin der Arteria iliaca communis sinistra an, den Verlauf der Vena iliaca communis sinistra nachalımend. In diese linke Kardinalvene mündet in Höhe der 'Teilungsstelle dor Aorta ein von rechts unten her kommendes bleistiftdickes Gefïss, das der Vena iliaca communis dextra entspricht und eine anostomotische Querverbindang $z$ wischen dem der. Vena iliaca communis sinistra ent. sprechenden Gefäss und dem entlang der Arteria iliaca communis dextra verlaufenden venösen Stamm darstellt. Diese eben genannte Querverbindung (Abb. 2, V. i. c. d.) verläuft von rechts unten her hinter der rechten Arteria iliaca communis nach links oben vor der linken Arteria iliaca communis.

Die Lungen sind müssig ausgenehnt, blutreich. Eine bräunliche Verfärbung des Parenchyms ist nicht nachweisbar.

Die Schilddrüse ist entsprechend gross. Der linke Nervus recurrens vagi schlingt sich um den Ductus Botalli, der rechte um den Aortenbogen.

Bauchhöhle: Das lange und fettreiche grosse Notz bedeckt die mässig geblïhten Darmschlingen und ist nicht wie in gewobbnlicher Weise mit dem Colon transversum verbunden. Letzteres ist zum grössten Teil fest an der Hinterwand fixiert, namentlich liegt es in seipen mittleren Teilen der Radix mesenterii fest an. Dás hintere Blatt des grossen Netzes taberquert das Colon transversum etwas medial von der Flexura coli dextra und wird 
dann mit etwas unregelmässigen Strängen und Verdickungen wenig unterhalb des Quercolon an der Radix mesenterii adhärent. Gegen die Flexura coli sinistra inseriert das bintere Netzblatt am Quercolon selbst, das letztere wird hier auch freier und gewinnt ein Mesocolon. - Das Ligamentum gastrolienale setzt anormalerweise in einer tiefen, an der Hilusfläche etwas bogenförmig, nahe der vorderen Kante vom oberen zum unteren Pol verlaufenden tiefen Inzisur der Milz an, so dass zwischen dieser Insertionslinie und dem Milzhilus ein fast hühnereigrosser Milzteil in die Bursa omentalis hineinragt. - Das Peritoneum ist überall glatt und glänzend. Die Harnblase ist fanstgross. Der Uterus ist entsprechend gross und frei beweglich.

Die $\mathrm{Milz}$ ist vergrössert, $14,5 \mathrm{~cm}$ lang, $7 \mathrm{~cm}$ breit und unregelmässig figuriert. Durch die unter "Situs" beschriebene anormale Insertion des Ligamentum gastrolienale ist sie in zwei ungleiche Halften geteilt; sie zeigt eine prismatische Gestalt, wobei eine Seitenfläche der Bursa omentalis zugewendet ist and die oben erwähnte Incisur der vorderen Kante des Prismas entspricht. Auf dem gegenüberliegenden Scheitel findet sich eine seichtere, längs verlaufene Incisur.

Die Leber ist entsprechend gross, ziemlich blutreich. Die Pfortader verläuft an normaler Stelle. Der Lobus Spigeli ist zungenartig nach unten verlängert und nur durch einen dünnen, etwa kleinfingerdicken Stiel mit dem iibrigen Parenchym verbunden.

Pankreas: Hat nicht die normale längliche Form, sondern liegt als ein etwas platter, $7 \mathrm{~cm}$ breiter, $5 \mathrm{~cm}$ langer, $2 \mathrm{~cm}$ dicker Körper in dem hufeisenförmigen Bogen des Duodenum.

Nieren: o. B.

Genitalien: Die Vagina ist eng, frei von Missbildungen, ebenso der Uterus. Die Ovarien sind entsprechend gross, nur wenig gefurcht.

Anatomische Diagnose:

Defectus congenitalis partis inferioris septi atriorum (ostiam primum persistens) et foramen ovale apertum. Ductus Cuvieri sinister, Vena jugularis primitiva sinistra et Vena cardinalis sinistra persistens, Vena cava inferior deficiens, Dextropositio arcus aortae. Deformatiolienis et pancreatis et fixatio anormalis coli transversi.

Wir haben uns im folgenden zun̈̈chst mit den oben beschriebenen Missbildungen des Herzens, insbesondere des Vorhofseptums, und ihren Folgen, sodann mit den Anomalien der grossen arteriellen und der grossen venösen Giefïisse $7 u$ beschäftigen.

Eine Störung in der normalen Entwicklung des Vorhofseptums kann zum Offenbleiben des Foramen ovale, zu De- 
lcklen des Vorhofseptums oder anderweitigen Anomalien führen. Betrachten wir den bei dem vorliegenden Fall bestehenden grossen Defekt im Vorhofseptum mit seinem nach unten concaven Rand, so müssen wir mit B or n (4) annehmen, dass es sich um eine abnorme Persistenz des Ostium primum handell, d. h. dass das Septum I nicht zur Verwachsung mit den Atrioventrikularlippen gekommen ist, sondern höher oben unter Erhaltung einer Kommunikationsöffnung zwischen beiden Vorhölen stehen geblieben ist. Die oben erwähnten knolligen Verdickungen auf dem First des Ventrikelseptums dürfen vielleicht als Residuen der medialen Endocardkissen gedeutet werden.

Mehr Inleresse als dieser Defekt im Vorhofseptum beansprucht der zweite kreisrunde, dem Foramen ovale entsprechende Defekt, der von einem aus fibrösen Fäden bestehenden, spinnennetzartigen Fadenwerk überlagert ist. Die Bildung von sogenannten abnormen Sehnenfïden im rechten Vorhol gehört zu den Seltenheiten. Die letzten eingehenden Untersuchungen über diese Anomalie stammen von $\mathrm{Ch}$ i a ri (6). Dieser beschreibt 11 Fïlle, bei denen sich netzartige Bildungen meisl von der Valvula Eustachii und der Valvula Thebesii zum T'uberculum Lóweri spannen und beutellörmig gegen das Ostium venosum dextrum herabhïngen. Unserem Fall ähnelt am meisten der Fall 8 von C hiari, bei dem sich - ausser einem der soeben beschriebenen Netzwerke - ein ,im hinteren Abschnitt. ler Fossa ovalis auf dem Limbus Vieussenii gelagertes zartes Netzwerk" vorfindet. Bei der mikroskopischen Untersuchung fand Chiari, dass die Fïden aus faserigem Bindegewebe bestanden, etliche aber auch Züge verzweigter Muskelfasern enthiellen. Chiari hiilt diese abnormen Sehnenfäden für Rfsiduen der rechten Sinusklappe oder auch des Septum spurium, je nachdem sie in der Gegend der Mündungen der Hohlvenen und des Sinus coronarius gelagert sind, oder aber im Bereiche des Limbus Vieussenii und der Fossa ovalis. 
Im vorliegenden Fall kann man, da die Netzbildung keinerlei Zusammenhang mit der Valvula Eustachii zeigt, das Foramen ovale ausserdem offen ist und also keine vollständige Utberlagerung durch das Septum spurium stattgefunden hat, die Fadenbildungen als Residuen des Septum spurium auffassen. Das gleiche gilt meiner Ansicht nach für den Fall 8 von $\mathrm{Chiari,}$ da ja die Entstehung des Limbus Vieussenii, auf dem sich hier das Netzwerk vorfindet, auf das Septum spurium zurückzuführen ist. Doch ist auch zu bedenken, dass möglicherweise die Fadenbildungen $z u$ gleicher Zeit mit dem Foramen ovale entstanden sein können, also aus dem Septum I stammen, und dass es in diesem Fall gar nicht zu einem Herabwachsen des Septum spurium gekommen ist. Andererseits spricht der Bestand eines Limbus Vieussenii beim vorliegenden Fall dafür, dass ein Herabwachsen des Septum spurium stattgefunden hat. - Einen weiteren Fall von einer Netzbildung, die oben an der rechten Seite der oberen Hohlvenenmündung, vorn am linken Umfang des Auriculareingangs haftet, erwähnt Preis z (32) (Fall XII). Preisz hält diese Membran ebenfalls für ein Uberbleibsel des Septum spurium.

In allen anderen mir in der Literatur bekannt gewordenen Fällen sind die Netzbildungen im rechten Vorhof wohl als Residuen der rechten Sinusklappe zu betrachten: so in den Fällen von $\mathrm{Chiari}$, ferner in den beiden Fällen, über die R'otitiansky (33) berichtet (Defekte der Scheidewände, B. 2, Fall 14 und 16), sowie in dem von $L$ oos er (25) beschriebenen Fall, bei dem eine grosse fadige Membran von den Mündungsstellen der Vena cava inferior und des Sinus coronarius cordis nach oben zum rechten Rand der Mündung der oberen Hohlvene zieht. Auch Ebbinghaus (8) deutet eine Netzbildung, die sich - bei gleichzeitigem Bestehen mehrerer Perforationsöffnungen in der Vorhofscheidewand - zwischen dem oberen Rand der Valvula Eustachii und den beiden Hohlvenenmündungen ausspannt, als Rest der Valvula venosa dextra. 
Mithin stellt das bei dem vorliegenden Fall bestehende fiidige Netz über dem Foramen ovale ein seltenes Vorkommnis dar.

Dif Spaltung des Aortenzipfels der Mitralis is $t$ bei gleichzeitig bestehendem Defekt in der Vorhofscheide. wand keine so seltene Erscheinung. Die normale Entwicklung der medialen Zipfel der venösen Ostien stellt sich etwa folgendermassen dar (vgl. Herxheimer [16]): das Ostium atrioventriculare commune rückt in die Mitte und wird dadurch, dass das vordere und das hintere Endocardkissen aufeinander zuwachsen und miteinander verlöten, in zwei, ein links und ein rechts gelegenes Ostium geschieden. So entstehen vier Endocardkissen, von denen die beiden medialen als Wülste in das rechte und linke Ostium atrioventriculare vorspringen. Sie liefern. die Grundlage für je eine mediale Zipfelklappe.

R uge (35) beschreibt ebenfalls zwei Fälle von Spaltung des Aortenzipfels bei gleichzeitig bestehendem Defekt des Vorhofseptums und führt die Spaltung des Zipfels darauf zurück, dass das Septum I die Endocardkissen nicht erreicht hat und die Verwachsung der beiden entsprechenden Wülste untereinander ausgeblieben ist. Auch im vorliegenden Fall ist zweifellos ein Zusammenhang vorhanden zwischen der Spaltung des Aortenzipfels: der Mitralis und dem mangelnden Herabwachsen des Septum I. - Ähnliche Fälle finden sich weiterhin bei $\mathrm{Ro}$ kit.ansky (33) (A 1, Fälle 2, 3 und 4, sowie A 2, Fälle 1-6); ferner beschreibt L or en z (26) einen Fall von Spaltung. des Aortenzipfels der Mitralis bei vollstïndigem Fehlen des Septum atriorum und kleinerem Defekt im hinteren oberen Teil des Septum ventriculorum. - Eine Insuffizienz der Mitralis dürte in unserem Falle, da sonst alle Klappensegel wohl ausgebildet sind, nicht bestanden haben, ist also auch für die Dilatation des rechten Herzens nicht verantwortlich zu machen. Im Gegensatz hierzu schreibt Rok'itansky (34) zu einigen 
seiner Fälle, dass „eine Spaltung des Aortenzipfels der Bicuspidalklappe von besonderer Bedeutung wird, indem sie durch die gesetzte Insuffizienz der Bicuspidalis die Erweiterung vom Lungenvenensack aus begünstigen und zugleich machen muss, dass die Aorta in der Entwicklung ihres Kalibers zurückbleibt". - Ob die im vorliegenden Fall bestehende Verkümmerung des medialen Tricuspidalsegels eine Tricuspidalinsuffizienz zur Folge gehabt hat, lässt sich nicht mit Sicherheit entscheiden. Es wäre sehr wohl möglich, dass das besonders gross und stark entwickelte vordere Tricuspidalsegel eine vollständige Schlussfähigkeit der Klappe bewirkt hat.

Wir sahen, dass am Herzen unseres Falles allein der rechte Ventrikel fast doppelt so gross ist, als die Faust der Leiche, während der linke Ventrikel bei der Ansicht von vorn ganz verschwindet und der Conus pulmonalis den grössten Teil der linken Herzgrenze ausmacht, dass ferner die Muskulatur des rechten Ventrikels erheblich stärker ist als die des linken, und dass schliesslich die Pulmonalis an ihrem Beginn mehrmals so stark ist als die Aorta, welch letztere nur einen Durchmesser von $2 \mathrm{~cm}$ besitzt. Worauf ist nun diese enorme Dilatation und Hypertrophie des rechten Herzens zuräckzuführen?

Eine Mitralinsuffizienz besteht nicht, auch ist es zweifelhaft, $a b$ die Enge der Aorta und die Weite des Pulmonalis auf eine ungleiche Teilung des Truncus arteriosus durch' das Septum trunci zurückzuführen ist. Es kann also die Erweiterung des rechten Ventrikels wohl nur so zustande gekommen sein, dass das Lungenvenenblut vom linken Vorhof aus seinen Weg - anstatt in die linke Kammer - zum grössten Teil durch den Septumdefekt in den rechten Vorhof und in die rechte Kammer genommen hat, so dass dort infolge der übergrossen Blutzufuhr zunächst eine Stauung entstanden ist. Eine eventuell hinżukommende Insuffizienz der Tricuspidalklappe würde die Dila- 
Lation und Hypertrophie des rechten Ventrikels, sowie die Lrweiterung der Pulmonalis begünstigt haben. Die Engigkeit der Aorta kann man dann als eine Folge der geringeren Blutfïllung und Inanspruchnahme des linken Ventrikels auffassen.

Auf die Tatsache, dass man bei bestehenden Defekten des Vorhofseptums hïufig grosse Weite der Pulmonalis und Engigkeit der Aorta, sowie einen weiten, dickwandigen Ventrikel findet, hat Marchand (27) hingewiesen. Ich verweise hier ausserdem auf den auf $\mathrm{S}$. 429 beschriebenen Fall von $\mathrm{Mar}$ chand, sowie auf den Fall von Berthel (S. 431), die gleichfalls beide Weite der Pulmonalis und Enge der Aorta bei gleichzeitig hestehenden Septumdefekten aufweisen. Bei Rokilansky (33) finden wir mehrere Fälle, die neben Septumdefekten und Spaltung des medialen Mitralzipfels eine überwiegende Weite der Lungenarterie zeigen (A 2, Fülle 2-5). Auch R uge (35) beschreibt einen Fall (Fall 5) von stark delektem Vorhofseptum und völlig mangelndem Kammerseptum; die Aorta misst hier nur $2 \mathrm{~cm}$, die Pulmonalis hingegen $8 \mathrm{~cm}$ in Umfang.

Die A orta ist im vorliegenden Fall nach rechts gekrümmt, die Abgangsfolge ihrer Äste ist: Carotis communis sinistra, Carotis communis dextra, Subclavia dextra. Die Subclavia sinistra hingegen entspringt von einem gabelförmig von der Aorta nach links oben abzweigenden Stamm, der sich in den Strang des Ductus Rotalli fortsetzt (vgl. S. 413).

Die im embryonalen Leben bestehenden 6 Kiemenbogenarterien unterliegen bekanntlich zum Teil einer Rückbildung. Wichtig für unsere Betrachtungen ist nur das Verhalten der vierten und fünften Kiemenbogenarterie. Beide entspringen aus dem Truncus arteriosus. Aus der vierten linken Kiemenbogenarterie ,wird die Aorta, aus der fünften linken der Ductus Botalli; etwa an der Vereinigungsstelle beider sprosst aus der Aorta die Arteria subclavia sinistra hervor. Die rechte vierte 
Kiemenbogenarterie wird zur Arteria subclavia dextra, während die fünfte rechte Kiemenbogenarterie vollständig obliteriert.

Bei diesen Umbildungen der Kiemenbogenarterien kommen mannigfache Anomalien vor. Beim vorliegenden Fall kommen wir zu folgendem Ergebnis: die Aorta ist aus der vierten rechten, anstatt aus der vierten linken Kiemenbogenarterie entstanden, dlaher nach rechts gekrümmt. Ihre beiden ersten Äste, die Carotiden, sind normalerweise aus den ventralen Verbindungsstücken der dritten und vierten Kiemenbogenarterie entstanden. Die Arteria subclavia dextra hingegen ist nicht eine persistierende Kiemenbogenarterie, sondern sprosst isoliert aus der Aorta hervor, ganz entsprechend dem Verhalten, wie es normalerweise die Arteria subclavia sinistra zeigt; hierdurch wird auch der Umstand erklärt, dass die Subclavia dextra und die Carotis dextra nicht - - wie gewöhnlich - aus einem gemeinsamen Stamm entspringen. Der fingerstarke, etwa $2 \mathrm{~cm}$ lange Stamm, der gabelförmig von der Ubergangsstelle des Arcus aortae in die Aorta descendens nach links oben abzweigt, dürfte eine Anlage der vierten linken Kiemenbogenarterie darstellen, also ein Rudiment der normal verlaufenden Aorta, das jedoch nur bis zur Einmündungsstelle des Ductus Botalli entwickelt ist und dann direkt in diesen übergeht. Der. Ductus Botalli ist nach links gewendet, also aus der fünften linken Kiemenbogenarterie entstanden. Die Arteria subclavia sinistra ist aus dem persistierenden Stück der vierten linken Kiemenbogenarterie hervorgesprosst. Es hat also hier weder rechts noch links die Umwandlung einer Kiemenbogenarterie in eine Arteria subclavia stattgefunden. Bezüglich des Rudimentes der linken Aorta und des Ursprungs der Arteria subclavia sinistra bei unserem Fall verweise ich auf die Figuren Nr. 109 und 112 im Handbuch der Gefässlehre von Henle (13), die ähnliche Verhältnisse zeigen.

In einem von Ghon (10) mitgeteilten Fall ist die Aorta nach rechts gekrümmt. Die Ursprungsfolge der grossen Gefässe 
ist: Arteria carotis communis sinistra, Arteria carotis communis dextra, Arteria subclavia dextra. Von der Pulmonalarterie zieht ein 1,3 cm langes, $0,4 \mathrm{~cm}$ im Durchmesser messendes Gefäss zum Arcus aortae (Ductus Botalli dexter). Von der Teilungsstelle der Pulmonalarterie entspringt ein für eine Sonde durchgängiges Gefäss (Ductus Botalli sinister), das gegen die linke Schlüsselbeinarterie hin zieht, nach einem Verlauf von $2 \mathrm{~cm}$ sich bulbusartig erweitert und dann in ein doppelt so weites Gefäss übergeht (Arteria subclavia sinistra). Im übrigen ist ein kleiner Defekt im vorderen oberen Teil des Ventrikelseptums vorhanden, Durchgängigkeit des Foramen ovale, Stenose des Conus pulmonalis und einige unbecleutende Venenanomalien. - Es existieren hier also zwei Ductus Botalli, die aus den beiden fünften Kiemenbogenarterien hervorgegangen sind. Die rechte Arteria subclavia verhält sich wie in unserem Fall. Von cler vierten linken Kiemenbogenarterie ist nichts erhalten gehlieben, als etwa die bulbusartige Erweiterung am Ende des linken Ductus Botalli, die wohl als ein Uberrest der ersteren gedeutet werden kann. Aus diesem Rudiment der normal verlaufenden Aorta ist die Arteria subclavia sinistra hervorgesprosst.

Ein von Gruber (12) beschriebener lall ist insotern erwähnenswert, als wir hier Ursprungsverhïltnisse der grossen Gefässe finden, die gegenüber den normalen gerade umgekehrt sind: Der Aortenbogen ist nach rechts gekrümmt. Die Astfolge der grossen Gefässe ist: Arteria subclavia sinistra und Arteria carotis communis sinistra, die aus einem gemeinsamen Stamm entspringen, dann die Arteria carotis communis dextra, am weitesten nach rechts die Arteria subclavia dextra. Der nach rechts gewendete Ductus Botalli ist weit offen. Das venöse System zeichnet sich durch Bestehen zweier primitiver Jugularvenen aus, von denen die rechte in den rechten, die linke in rlen linken Vorhof mündet. Beide Vorhöfe kommunizieren durch 
eine weite Offnung. - $\mathrm{Da}$ die linke Carotis und die linke Schlüsselbeinarterie hier von einem Stamm entspringen, stellt die letztere eine vierte linke Kiemenbogenarterie dar. Sie bildet mit der Arteria carotis communis sinistra, die ja aus dem ventralen Verbindungsstück zwischen dritter und vierter Kiemenbogenarterie entsteht, eine gemeinsame Arteria anonyma, derselbe Vorgang, wie er normalerweise rechts stattfindet. Der Ductus Botalli ist aus der rechten fünften Kiemenbogenarteric hervorgegangen, und die rechte Schlüsselbeinarterie ist aus der Aorta hervorgesprosst, entsprechend dem normalen Verhalten der linken Arteria subclavia. Es ist also hier - im Gegensatz zum vorliegenden Fall - die Arteria subclavia sinistra direkt aus einer Kiemenbogenarterie hervorgegangen.

Bei einem Fall von Rechtskrümmung der Aorta, über den Gross (11) berichtet, finden wir folgenden eigenartigen $\mathrm{Ab}$ gangsmodus der grossen Gefässe: zunächst. zweigen die beiden Carotiden $a b$, zuerst die linke, dann die rechte. Weiterbin an der Übergangsstelle des Arcus aortae in die Aorta descendens die Arteria subclavia dextra, und dann erst die Arteria subclavia sinistra, die nach links umbiegt und hinter dem Ösophaguis zur linken oberen Extremität zieht. - Es ist hier also die vierte rechte Kiemenbogenarterie zur Aorta geworden, die vierte linke Kiemenbogenarterie hat sich nicht wie im Fall Gruber (12) zur Arteria subclavia sinistra umgebildet, sondern ist verödet. Beide Schlüsselbeinarterien dürften aus der Aorta hervorgesprosst sein. Einen ganz ähnlichen Fall beschreibt Schwegel (37). Beiläufig möchte ich noch einen Fall von Fle is chmann (9) erwähnen, der einen der ersten publizierten derartigen Fälle darstellt - ex stammt aus dem Jahre 1815 -, und der insofern interessant ist, als er genau das Umgekehrte vom Fall Gross darstellt: Die Aorta ist nach links gekrümmt; die Reihenfolge ihrer Äste ist: A. carotis dextra, A. carotis sinistra, A. subclavia sinistra, A. subclavia 
dextra, welch letztere hinter dem Osophagus nach rechts verläuft. - Schliesslich weise ich noch auf die Übersicht und die grosse Anzahl von Fällen von Rechtslage der Aorta hin, die Henle (13) in seinem Handbuch der Gefässlehre bringt.

Der Befund am venösen System des vorliegenden Falles sei nochmals kurz skizziert:

Links neben der Halswirbelsäule verläuft symmetrisch zur normalen Vena cava superior ein venöser Stamm, der jedoch nicht in eine mit der Vena cava superior anastomosierende Vena anonyma ausläuft, sondern in den Sinus coronarius cordis mündet. In diesen Stamm ergiesst sich von hinten und oben her ein starkes venöses Gefäss (Abb. 2, V. c. s.), das aus dem Becken links neben der Wirbelsäule emporsteigt. Dieses Gefäss gibt in der Höhe der Nieren nach links die linke Nierenvene, nach rechts eine breite, die Aorta an ihrer Hinterfläche kreuzende Querverbindung ab, die sich wiederum teilt: einerseits in die rechte Nierenvene, andererseits in einen Stamm, der nach abwärts zieht und sich dem Verlauf der rechten Arteria iliaca communis anschliesst (Abb. 2, V. c. d.). Zwischen diesem letztgenannten Stamm und dem links neben der Wirbelsäule verlaufenden Hauptgefäss, das sich nach unten weiterhin dem Verlauf der linken Arteria iliaca communis anschliesst, besteht eine anastomotische Querverbindung. Die Vena cava inferior fehlt vollkommen, die Lebervene mündet direkt in den rechten Vorhof.

Vergleichen wir diese Bildungsanomalien mit der embryonalen Entwicklung, so handelt es sich im vorliegenden Fall um eine Persistenz embryonaler Verhältnisse. Im Gebiet der oberen Hohlvene ist die Bildung der Vena anonyma ausgeblieben, es persistiert die linke primitive Jugularvene, deren Endstück zum Sinus coronarius cordis geworden ist. Wir können also die Bildung als Persistenz beider primitiver Jugularvenen bezeichnen. Weiter- 
hin ist die Bildung der unteren Hohlvene ausgeblieben. Vielmehr persistiert der caudale Abschnitt der rech ten $\mathrm{Car}$ dinalvene, der sich normalerweise an der Bildung der unteren Hohlvene beteiligt, im vorliegenden Fall als ein selbständiger Stamm (siehe Abb. 2, V. c. d.), der an der lateralen Seite der Arteria iliaca communis dextra heraufsteigend, sich mit der rechten Nierenvene zu einem dicken Stamm vereinigt, welch letzterer die Aorta an ihrer hinteren Fläche überquert und in die linke Cardinalvene mündet. Diese Deutung des vorliegenden Falles hat bereits Herzog in seiner vorläufigen Mitteilung (vgl. S. 411) gegeben; auch V e r s è (38) hat in einem ähnlichen Fall (vgl. S. 428) von Persistenz der caudalen Abschnitte der Cardinalvenen gesprochen. - Der craniale Abschnitt der rechten Cardinalvene hingegen hat sich bei unserem Fall vermutlich zur Vena azygos umgewandelt. Das starke Gefäss aber, das links neben der Wirbelsäule aufwärts verläuft, ist als die persistierende linke Cardinalvene zu deuten. Sie sammelt das Blut aus den unteren Extremitäten und dem Becken, nimmt die Nierenvenen auf und mündet schliesslich in die linke primitive Jugularvene, mit dieser einen Ductus $\mathrm{Cuvieri}$ bildend. Wir haben also vor uns eine unvollkommene Persistenz der rechten, eine vollkommene Persistenz der linken Cardinalvene, welch letztere die Funktion der Vena cava inferior übernommen hat. Auch der Verlauf der Lebervene ist der gleiche, wie unter embryonalen Verhältnissen.

Die von der linken Cardinalvene in Höhe der rechten Niere nach rechts abzweigende und sich dann in rechte Nierenvene und rechte Cardinalvene teilende Querverbindung kreuzt die Aorta an ihrer Hinterfläche, während normalerweise die linke Nierenvene vor der Aorta nach links hinüberzieht. $\mathrm{Obexn}$. dorfer (30) beschreibt einen Fall von Persistenz einer Cardinalvene mit einer ähnlichen Querverbindung; diese verläuft vor der Aorta, auch ist hier die Vena cava inferior voll ent- 
wickelt und die linke Cardinalvene persistiert nur bis hinauf zu der Querverbindung, während im vorliegenden Fall die linke Cardinalvene in ganzer Länge, die rechte aber nur bis hinauf zu der erwähnten Querverbindung persistiert. Wir dürfen mit Oberndorfer (30) annehmen, dass ,diese Querverbindung die ursprüngliche Queranastomose zwischen der unpaar angelegten Vena cava inferior und der Vena cardinalis sinistra darstellt. Diese Anastomose leitet normalerweise das Schwächerwerden der Vena cardinalis sinistra ein, die dann später völlig ausser Funktion gesetzt wird und verödet".

Von besonderem Interesse ist bei unserem Fall noch die Bildung der Venae ilia cae communes und ihr Verhältnis zum caudalen Abschnitt der rechten Cardinalvene.

Normalerweise geht die Vena hypogastrica sowie die Vena iliaca externa aus den untersten segmentalen Zweigen der entsprechenden Cardinalvene hervor. Nach Rückbildung der linken Cardinalvene gewinnen die Vena hypogastrica sinistra und die Vena iliaca externa sinistra den Anschluss an die rechte Cardinalvene - und damit an die untere Hohlvene - durch Ausweitung einer Anastomose mit der rechten Cardinalvene; diese Anastomose wird später zur Vena iliaca communis sinistra. Diese Verhältnisse zeigen besonders deutlich die Figuren im Lehrbuch der Anatomie von Langer-Toldt, S. 537.

Bei uns ist nun offenbar das Ungekehrte eingetreten: es hat sich von der rechten Cardinalvene aus eine Anastomose (Abb. 2, V. i. c. d.) nach links oben gebildet, die in die zusammen mit der linken Arteria iliaca communis verlaufende Vena cardinalis sinistra mündet. Die rechte Cardinalvene führt die Hauptmenge des Blutes aus der rechten Beckenhälfte und der rechten unteren Extremität, so dass die erwähnte Anastomose, die Anlage der sonstigen Vena iliaca communis dextra, gar nicht zu ihrer vollen Entwicklung gekommen ist. - Ferner ist beim vorliegenden Fall bemerkenswert das Verhältnis der 
Venae iliacae communes zu den Arteriae iliacae communes. Während normalerweise die rechte Arteria iliaca communis die Vena cava inferior an ihrer Vorderfläche, und zwar an der Teilungsstelle in die beiden Venae iliacae communes, überkreuzt, so dass weiterhin die beiden Venae iliacae communes an der' medialen Seite der entsprechenden Arterien verlaufen, stellen sich bei unserem Fall diese Verhältnisse so dar, dass die linke Vena iliaca communis als die direkte Fortsetzung der linken Cardinalvene an der lateralen Seite der Arteria iliaca communis sinistra verläuft; die oben erwähnte Anastomose aber, die die Vena iliaca communis dextra vertritt, verläuft zunächst hinter der rechten Arteria iliaca communis, dann vor der linken Arteria iliaca communis, um schliesslich in die linke Cardinalvene zu münden, die an der lateralen Seite der Arteria iliaca communis sinistra abwärts zieht.

Schliesslich ist am Gefüsssystem des vorliegenden Falles noch bemerkenswert der Verlauf der Venae spermaticae internae. Während normalerweise die linke Vena spermatica interna in die linke Nierenvene mündet, die rechte hingegen in die Vena cava inferior, haben wir hier das umgekehrte Bild vor uns: die linke Vena spermatica interna mündet direkt in die linke Cardinalvene, die rechte hingegen in die entsprechende Nierenvene (vgl. hierzu auch den auf S. 429 erwähnten Fall von D o r sc h [7]). Die Tatsache, dass bei unserem Fall - wie auch aus der Beschreibung von Herzog hervorgeht - beide Venae spermaticae voll ausgebildet sind und neben ihnen die linke Cardinalvene vollständig, die rechte in ihrem infrarenalen Abschnitt persistieren, spricht gegen die Annahme, dass die Venae spermaticae internae entwicklungsgeschichtlich aus den Cardinalvenen hervorgegangen seien. Dasselbe gilt für den Fall Benöhr (2) (vgl. S. 430), bei dem die Venae spermaticae internae unabhängig von der Cardinalvene in die entsprechenden Nierenvenen münden. V ersé (38) be- 
schreibt einen Fall (vgl. S. 425) von kongenitaler Pulmonalstenose, breitem Defekt des Septum ventriculorum und offenem Foramen ovale, bei dem zwei Cardinalvenen in ihrem infrarenalen Abschnitt persistieren, sich jedoch in Höhe der Nieren zu der normal verlaufenden Vena cava inferior vereinigen. Die Venae spermaticae internae münden hier beiderseits in die entsprechenden Cardinalvenen.

Es seien nun noch einige lälle von Anomalien des Venensystems kurz beschrieben, die mit dem unseren eine mehr oder minder weilgehende Ähnlichkeit haben.

Loch te (24) beschreibt einen Fall von Situs transversus der Organe der Brust- und Bauchhöhle. Die Vena cava inferior fehlt vollkommen und ist ersetzt durch die persistierende linke Cardinalvene. Diese mündet in eine linke primitive Jugularvene, mit letzterer einen Ductus Cuvieri bildend, der sich in den linken Vorhof einsenkt. Dic Lebervene mündet ebenfalls direkt in den linken Vorhof. Im Septum ventriculorum besteht eine zehnpfennigstückgrosse öffnung, die Pars membranacea fehlt vollständig. Die Aorta, die aus dem rechten Ventrikel entspringt, ist nach rechts gekrümmt. Die Milz ist durch eine vom vorderen Rande in das Parenchym eindringende Incisur in zwei Teile gespalten; in der Tiefe der Incisur haftet ein Teil des grossen Netzes. - Wir haben also hier - abgesehen von dem Situs transversus - ganz ähnliche Verhältnisse, wie beim vorliegenden Fall. Es sei bei dieser Gelegenheit darauf hingewiesen, dass - wie $\mathrm{M} \mathrm{arch}$ and (27) betont - Lageanomalien der Brust- und Baucheingeweide bei gleichzeitig bestehenden Anomalien des Venensystems kein ganz seltener Befund sind. Coste und Kölliker (erwühnt bei Marchand [28]) führen diese Entwicklungsstörungen - Lageanomalien der Eingeweide und Missbildungen des venösen Systems - auf eine Störung in der Entwicklung der Dottervenen und der Lageverhältnisse der Venen bei der Drehung des Magens zurück. 
Im Falle von Marchand (28) handelt es sich um Persistenz der rechten Cardinalvene nebst anderweitigen Anomalien: Das Herz ist in den Ventrikeln nicht vergrössert. Das Septum atriorum fehlt vollst:̈ndig, so dass nur ein gemeinsamer Vorhof vorhanden ist. Im oberen hinteren Teil des Ventrikelseptums besteht ein grosser halbmondförmiger Defekt. Die Arteria pulmonalis ist sehr weit, während der Conus arteriosus sinister eng, spaltförmig ist und die Aorta am Isthmus eine Stenose aufweist. Ausser der normalen Vena cava superior findet sich eine linke primitive Jugularvene. Die Vena anonyma lehlt. An der Einmündungsstelle der linken primitiven Jugularvene in den rechten Vorhof ist die Wand der ersteren durchbrochen, so dass ein Teil des Blutes in den Sinus curonarius fliesst, ein Teil aber durch die Durchbruchsstelle direkt in den rechten Vorhof. Die Lebervenen münden direkt in den rechten Vorhof. An Stelle der fehlenden Vena cava inferior persistiert die rechte Cardinalvene, die rechts neben der Aorta durch den Hiatus aorticus verläuft und sich in die rechte obere Hohlvene einsenkt. Die Valvulae Thebesii und Eustachii fehlen. Die Organe der Brust- und Bauchhöhle zeigen partiellen Situs inversus, Magen und Milz sind nach rechts unterhalb der Leber verlagert, Dünn- und Dickdarm weisen erhebliche Anomalien auf. Die Milz ist in zwei Teile gespalten, in der Furche zwischen beiden haftet das grosse Netz. - Hier hat also die rechte Cardinalvene die Funktion der unteren Hohlvene übernommen und bildet mit der rechten oberen Hohlvene (persistierenden rechten primitiven Jugularvene) einen Ductus Cuvieri. Uber die Enge der Aorta und Weite der Arteria pulmonalis vergleiche das aul Seite 420 Gesagte.

Weiterhin berichtet Dorsch (7) über einen Fall, der mit dem unseren weitgehende Ähnlichkeit hat; die Vena cava inferior wird durch die linke Cardinalvene vertreten, die sich in eine primitive linke Jugularvene ergiesst. Die linke Vena sper- 
matica interna mündet direkt in die Cardinalvene, die rechte hingegen in die rechte Nierenvene. Die Lebervenen öffnen sich direkt in den rechten Vorhof. - Beiläufig sei hier erwähnt; dass auch bei normal angelegter Vena cava inferior die direkte Einmündung der Lebervenen ins Herz vorkommt, wie die Fälle von Rokitansky (33) und Kaydi (18) beweisen.

Fernerhin sind in der Literatur Fälle bekannt, in denen das die Vena cava inferior vertretende Gefäss seine Entstehung sowohl der rechten als auch der linken Cardinalvene verdankt. Wir finden bei Dorsch (7) einen derartigen Fall erwähnt, der überhaupt einen der ältesten in der Literatur bekannt gewordenen Fälle derartiger Venenanomalien darstellt: Die Dissertation von Gurlt „De venarum difformitatibus; Vratislaviae 1819". Es handelt sich hier um die Persistenz einer Vena cardinalis bei fehlender unterer Hohlvene. Die Cardinalvene läuft zunächst links von der Wirbelsäule nach' aufwärts, wendet sich vor dem 9 . und 8. Brustwirbel nach rechts, übersteigt den rechten Bronchus und senkt sich in die Vena cava superior ein. Die Lebervenen münden direkt in den rechten Vorhof. - Offenbar ist an der Bildung dieses Gefässes nicht nur die rechte, sondern auch - in dem abwärts vom 9. Brustwirbel gelegenen Abschnitt - die linke Cardinalvene beteiligt gewesen.

Ein ähnlicher Fall ist der von Benöhr (2). Hier verläuft die Cardinalvene zunächst rechts von der Wirbelsäule, wendet sich in der Höhe der Nieren nach links, um sich dann oberhalb des $Z$ werchfells wieder auf die rechte Seite zu begeben, den rechten Bronchus zu übersteigen und in die obere Hohlvene einzumünden. Die Venae spermaticae internae münden hier beide in die gleichseitigen Nierenvenen. Hier dürfte also an der Bildung des die untere Hohlvene vertretenden Gefässes im wesentlichen die rechte, in dem Abschnitt zwischen Nierenvenen und $Z$ werchfell jedoch auch die linke Cardinalvene beteiligt sein. 
Ein weiterer derartiger Fall findet sich bei Ko $11 \mathrm{man} \mathrm{n}(22)$, wo zunächst zwei Cardinalvenen bestehen, die sich im Hiatus aorticus des Zwerchfells zu einem Gefäss vereinigen, das zunächst links, dann rech's von der Wirbelsäule emporzieht fernerhin bei C. F. Krause (23) und otto (31).

In den beiden Fällen, die Kipper (19) und Herholdt (14) beschreiben, ist ebenfalls die fehlende Vena cava inferior durch eine persistierende Cardinalvene ersetzt. Bei beiden besteht mehr oder minder vollkommener Situs inversus der Brustund Baucheingeweide, und es ist besonders interessant, dass im einen Fall die rechte Cardinalvene, die die untere Hohlvene vertritt, nach links transponiert ist und in die links gelegene obere Hohlvene mündet, im anderen Fall die nach rechts transponierte - also rechts von der Wirbelsäule verlaufende linke Cardinalvene in die links gelegene Vena cava superior mündet. Bei dem Fall von $\mathrm{K}$ i p per ist noch erwähnenswert, dass die Milz durch eine Incisur, in der das grosse Netz inseriert, in zwei Teile gespalten ist.

Einen Fall von Persistenz beider Cardinalvenen beschreitit Martin (29): Beide Venen münden in die entsprechenden primitiven Jugularvenen und bilden mit ihnen die Ductus $\mathrm{Cu}$ vieri. Ähnliche Verhältnisse treffen wir in dem Fall von Willige (40) an. Auch sei hier auf die sehr instruktive Abbildung Nr. 575 des Handatlasses von Kollmann (21) hingewiesen, die die Persistenz beider Cardinalvenen darstellt. Von den sonstigen, in den Rahmen dieser Betrachtungen gehörigen Fällen erwähne ich noch den von Berthel (3), der sich durch Persistenz einer linken Cardinalvene, offenes Foramen ovale, Enge der Aorta und Weite der Arteria pulmonalis auszeichnet, sowie die weniger genau beschriebenen Fälle von Virchow (39), Klob (20), Bujalski (5), Whinnie (41) und d'Alton (1).

Schliesslich sei nochmals an die verschiedenartigen Miss- 
bildungen erinnert, die die Bauch e ing e w ei de des vorliegenden Falles betreffen. Das Quercolon ist fest an der hinteren Bauchwand fixiert, das hintere Blatt des grossen Netzes ist fast in seiner ganzen Breite mit dem Mesenterium und der Radix mesenterii verwachsen. An der Leber weist der S pi gel sche Lappen eine zungenartige Verlängerung auf, während das Pancreas eine anormale, plattrundliche Form hat. Die Teilung der Milz in zwei - wie wir dies bei unserem Fall beobachten - oder mehrere Teile ist, wie Marchand (27) betont, ein bei Anomalien des Venensystems häufig vorkommender Befund. Die Milz ist dabei oft durch eine Incisur, in der das grosse Netz inseriert, unvollkommen geteilt. Vergleiche dazu die oben erwähnten Fälle von Lochte (S. 428), Mar chand. (S. 429) und Kipper (S. 431).

Über die causale Genese der beim vorliegenden Fall bestehenden Missbildungen etwas Bestimmtes auszusagen, dürfte kaum möglich sein. Ich schliesse mich hierin der Ansichl Schwalbes (36) an: „Es gibt Hemmungsbildungen, besonders solche, die als Persistenz fötaler normaler Einrichtungen im extrauterinen Leben erscheinen, bei welchen eine Erklïrung, eine Frage nach der Ursache zunächst aussichtslos erscheint (offenes Foramen ovale etc.)." Auch die causale Abhängigkeit der einzelnen Anomalien untereinander dürfte kaum anzunehmen sein.

Auch über die Entstehungszeit der Missbildungen unseres Falles lässt sich etwas Bestimmtes nicht sagen. Nach Born (4), der die Entwicklung des Herzens an Kaninchenembryonen studiert hat, ist die erste Anlage des Septum I beim Embryo von $1,7 \mathrm{~mm}$ Kopflänge (10. Tag nach der Begattung) zu finden; voll ausgebildet ist es beim Embryo von 2,5-2,7 $\mathrm{mm}$ Kopflänge. In dieser Zeit ist das Septum I zu einem dünnen halbmondförmigen Kamm ausgewachsen, dessen freier Rand die obere Begrenzung des Ostium I bildet. Es ist damit der 
Zustand erreicht, den wir als dauernd bei unserem Fall vorfinden. Das Ostium I verkleinert sich weiterhin unter Annäherung des Randes des Septum I an die Endocardkissen und ist schliesslich bei Embryonen von 5,5-6 $\mathrm{mm}$ Kopflänge verschwunden. Die Verschmelzung der einander zugewandten Flächen des Endocardkissen ist beim Embryo von $6 \mathrm{~mm}$ Kopflänge (der 14. Tag) beendet. Bei einem menschlichen Embryo von $5 \mathrm{~mm}$ Kopflänge fand $\mathrm{B}$ orn das Ostium I noch offen, etwa gleichgross wie das 0stium II, bei $6 \mathrm{~mm}$ Kopflänge hingegen verschlossen. Wir können also mit einiger Wahrscheinlichkeit als Entstehungszeit des beim vorliegenden Fall bestehenden Septumdefektes etwa die Zeit annehmen, bevor der Embryo eine Kopflänge von 3-6 mm (12. bis 14. Tag) hatte.

Die Ausbildung der Vena cava inferior beginnt nach $\mathrm{Hoch}$. stetter (17) am 12. Tag der embryonalen Entwicklung; zu dieser Zeit erweitern sich die zwischen unterer Hohlvene und den Cardinalvenen bestehenden Anastomosen und die untere Hohlvene beginnt den Abfluss des Blutes nach dem Herzen zu übernehmen. Gegen Mitte des 13. Tages beginnt die teilweise Obliteration der Cardinalvenen. Wenn wir mit 0 bernd orfer (30) die bei unserem Fall bestehende Querverbindung (vgl. S. 426) als ein Uberbleibsel der zwischen unterer Hohlvene und linker Cardinalvene im Embryonalleben bestehenden Anastomose deuten, so können wir als Entstehungszeit der Missbildung etwa den 12.-13. Tag des Embryonallebens mutmassen, mithin die gleiche Zeit, zu der der Septumdefekt entstanden sein dürfte. Es ist also auch zeitlich eine Abhängigkeit der einzelnen Bildungsfehler voneinander nicht anzunehmen.

Zum Schluss sei mir gestattet, Herrn Prof. Herzog für seine Unterstützung bei der Abfassung der Arbeit meinen Dank auszusprechen. 


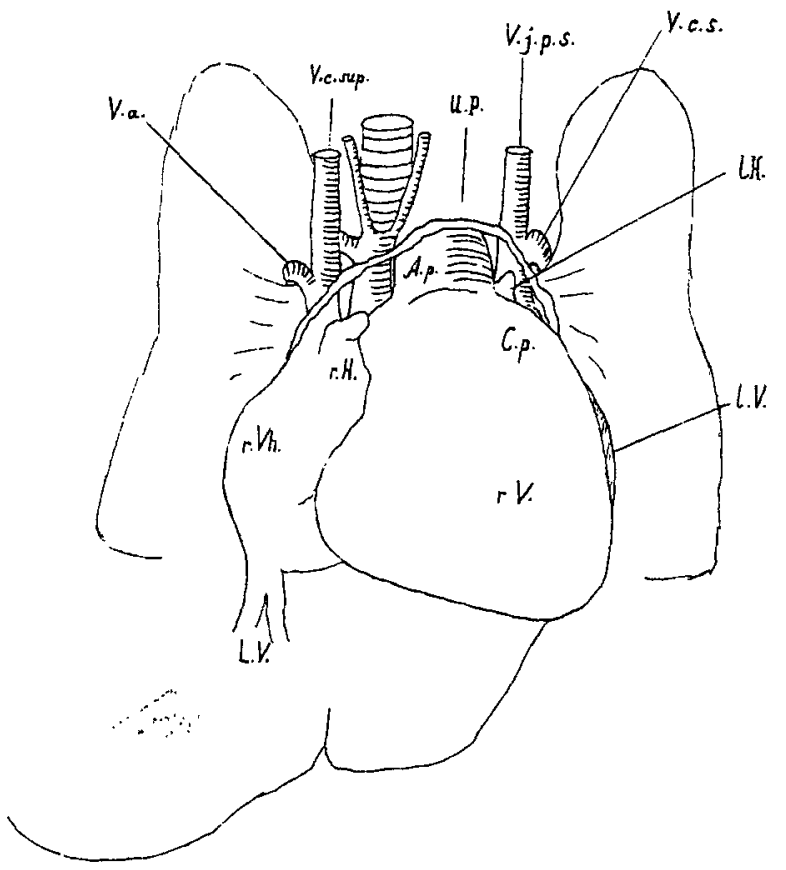

Abb. 1.

Herz von vorn.

C. p. Conus pulmonalis.

l. H. linkes Herzolur.

r. H. rechtes Herzohr.

r. V. rechter Ventrikel.

l. V. linker Ventrikel.

r. Vh. rechter Vorhof.

V.j.p.s. Vena jugularis primitiva sinistra.
V.c.s. Vena cardinalis sinistra.

V.c.sup. Vena cava superior.

V. a. Vena azygos.

L. V. Lebervenen.

U.P. Umschlagsfalte des Pericards.

A. p. Arteria pulmonalis. 


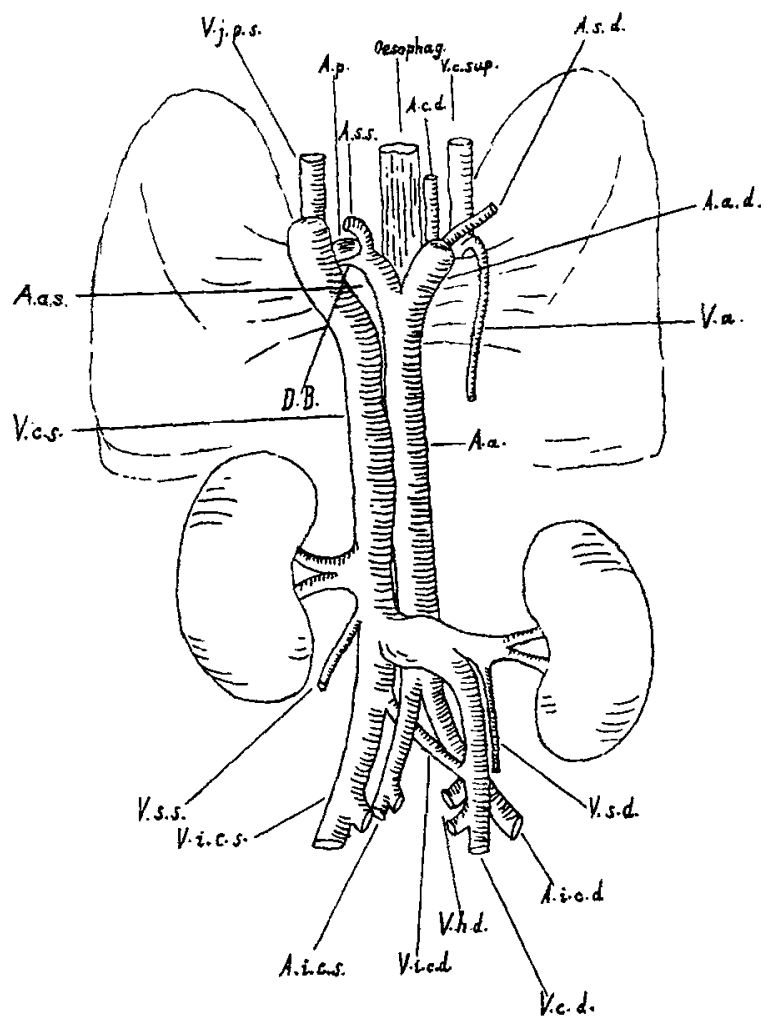

$\triangle b b .2$.

Herz und grosse Gefässe von hinten.

A. p. Arteria pulmonalis. V.c. sup. Vena cava superior.

A.s.s. Arteria subclavia sinistra. V.a. Vena azygos.

A. s. d. Arteria subclavia dextra. V.c.s. Vena cardinalis sinistra.

A.c.d. Arteria carotis comm. dextra. V.c.d. Vena cardinalis dextra.

A. a. d. Arcus aortae dexter. V.s. s. Vena spermatica sinistra.

A. a.s. Arcus aortae sinister. V.s.d. Vena spermatica dextra.

A. 2. Aorta abdominalis. V.i.c.s. Vena iliaca comm. sinistra.

A.i.c.s. Arteria iliaca comm. sinistra. V.i.c. d. Vena ilinca comm. dextra.

A. i. c. d. Arteria iliaca comm. dextra. V.h.d. Vasa hypogastrica dextra.

V.j.p.s. Vena jugularis primitiva D. B. Ductus Botalli. sinistra. 


\section{Literaturverzeichnis.}

1. d'Alton, zit. bei Gruber, Archiv f. Anatomie u. Pbysiologie. 1865.

2. Ben $8 \mathrm{hr}$, Inaug.-Diss. Kiel 1904.

3. Berthel, Inaug.-Diss. Munchen 1901.

4. Born, Archiv f. mikroskop. Anatomie. Bd. 33. 1889.

5. Bujalski, zit. bei Gruber ef. 1 .

6. Chiari, Zieglers Beiträge. Bd. 22. 1897.

7. Dorsch, Arztl. Intelligenzblatt. 1858.

8. Ebbinghau s, Manchener med. Wochenschrift. Nr. 18. 1904.

9. Fleis ch $\mathrm{m}$ a $\mathrm{n}$, Leicheneroffnungen. 1815 .

10. Ghon, Verhandlungen der deutschen pathol. Gesellschaft. 1908.

11. Gross, Zieglers Beiträge. Bd. 37.

12. Gruber, Prager Vierteljahrsschrift f. prakt. Heilkunde. Bd. 9. 1846.

13. Henle, Handbuch der Gefässlehre. 2. Aufl.

14. Herboldt, Bescriv. over et mennesk. Misfoster, Kjobenhavn. 1828.

15. Herzog, Mänch. med. Wochenschr. 1913. Nr. 37. S. 2090.

16. Herxheimer, Kapitel "Missbildungen des Herzens" in Schwalbe, Morphologie der Missbildungen. 1910.

17. Hochstetter, Morphol. Juhrbach. Bd. 20. 1893.

18. Kay di, Österr. med. Jahrbuch. 1881.

19. Ki p per, Inaug.-Diss. Marburg 1896.

20. Klob, Zeitschrift d. Gesellschaft d. Ärzte zu Wien. 1859.

21. Kollmann, Handatlas der Entwicklangsgeschichte d. Menschen. Bd. 2. 1907.

22. Derselbe, Anatom. Anzeiger. 1893.

23. Krause, C. F. Handbuch d. menschl. Anatomie. Bd. 3. 1838.

24. Lochte, Arbeiten a. d. patholog. Institut der Universität Leipzig. Bd. 3. 1899.

25. L o os e r, Inaug.-Diss. Zürich 1902.

26. Lorenz, Med. Jahrbuch d. K. u. K. Gesellschaft der Ärzte. Wien 1880.

27. Marchand, Artikel, Missbildungen" in Eulenburgs Realenzyklopädie Bd. 9. 1910.

28. Derselbe; Ahlfelds Berichte und Arbeiten aus der geburtshilfl. gynäkol. Klinik zu Giessen. 1881/82.

29. Martin, Monatsschrift für Geburṫkunde. Bd. 20. 1862.

30. O bernd orfer, Münchener med. Wochenschrift. Nr. 10. 1903.

31. Otto, Seltene Beobachtungen. 1824. 
32. Preisz, Zieglers Beitrăge. Bd. 7. 1890.

33. Rakitansky, Die Defekte der Scheidewănde des Herzens. 1875.

34. Derselbe, Handbuch der Anatomie. Bd. 2.

35. Ruge, Virch ow s Archiv. Bd. 126. 1891.

36. Schwalbe, Morphologie d. Missbildungen des Menschen u. d. Tiere. I. Teil. S. 179. Jena 1906.

37. Schwege l, Vierteljahrsschrift f. prakt. Heikunde. 1859.

38. Versé, Münchener med. Wochenschrift. Nr. 15. 1918.

39. Virchow, Archiv f. patholog. Anatomie u. Physiologie. 1861.

40. Willige, Inaug.-Diss. Göttingen 1904.

41. Whinnie, zit. bei Gruber of. 1 . 